sequences will be situated at the $5^{\prime}$ end of the polycistronic transcript (band $\mathrm{X}$ ).

The two other tRNA precursor bands ( $\mathrm{Y}$ and $\mathrm{Z}$ ) are about the size of $23 \mathrm{~S}$ rRNA $(3.2 \mathrm{~kb})$. Since RNA bands $Y$ and $Z$ contain the same promoter-proximal tRNA sequences as the longer RNA band $X$, their different size must be due to missing sequences at the $3^{\prime}$ end. This may be the result of an earlier specific termination of transcription. We cannot completely rule out, however, the possibility that bands $\mathrm{Y}$ and $\mathrm{Z}$ are derived from band $X$ by traces of processing nucleases which might be present in our RNA polymerase preparations.

The tRNA polycistronic precursor (band $\mathrm{X}$ ) is long enough to include the sequences of the two stable RNA species located at the end of tRNA operon (Fig. 1). Yet, we did not identify these RNA species among the products of $S-100$ processing of band $\mathrm{X}$ or of unfractionated mixture of T4 DNA transcripts. Although some bands of the expected molecular weight have been observed, they were not reproducible and we believe that these RNA species are not completely stable under the processing conditions used.

The isolation of the polycistronic T4 tRNA precursor makes the T4 tRNA operon a promising system for the study of the mechanism of T4 gene expression and the maturation of tRNA.

We thank Drs J. Abelson and K. Fukada for sending us the $\mathrm{T} 4 \mathrm{psu}_{\mathrm{b}}{ }^{-\Delta}$ mutants.

Alexander Goldfarb

EDNA SEAMAN*

ViOLET DANIEL

Department of Biochemistry,

Weizmann Institute of Science,

Rehovot. Israel

Received 31 January; accepted 31 March 1978.

*Permanent address: Department of Biology, University of Massachusetts, Boston Massachusetts.

. O'Farrell, P. Z. \& Gold, L. M. J. biol. Chem. 248, 5502-5511; 5512-5519 (1973). 2. Wood, W. B. \& Revel, H. R. Bact. Rev. 40,847-868(1976).

Abelson, J.et al. Brook haven Symp. Biol. 26, 77-88 (1974).

Guthric, C.et al. Brookhaven Symp. Biol. 26, 106-123(1974).

Wilson, J. H., Kim, J. S. \& Abelson, J. J. molec. Biol. 71, 547-556 (1972).

Nierlich, D. P., Lamfrom, H., Sarabhai, A. \& Abelson, J. Proc. natn. Acad. Sci. U.S.A.70, 179-182(1973)

. Kaplan, D. A. \& Nierlich, D. P. J.biol. Chem. 250,934-938 (1975).

Gold, L. M. \& Schweiger, M.J.biol. Chem. 245, 2255-2258(1970).

9. Black, L. W. \& Gold, L. M. J. molec. Biol. 60,365-388 (1971).

10. Scherberg, N. H., Guha, A. Hsu, W. -T. \& Weiss, S. B. Biochem. biophys. Res. Commun. 40,919-924 (1970).

11. Brody, E. N., Gold, L. M. \& Black, L. W. J. molec. Biol. 60, 389-393 (1971). 12. Black, L. W. Virology $60,166-179(1974)$.

13. Velten, J., Fukada, K. \& A belson, J. Gene 1,93-106 (1976).

14. Peacock, A. C. \& Dingman, W. C. Biochemistry 7, 668-674(1968).

15. Khesin, R. B., Bogdanova, E. S., Goldfarb, A. D. \& Zograff, Y. N. Molec. gen

16. Burgess, R. R. \& Jendrisak, J. J. Biochemistry 14, 4634-4638 (1975).

\section{Unusual segmental flexibility in a region of tobacco mosaic virus coat protein}

AsSembly of tobacco mosaic virus (TMV) is known to occur by threading in the RNA on the inner side of the growing hollow rodlet of viral coat protein, and is thought to involve a preassembled double disk as an intermediate ${ }^{1-3}$. Given the structure of the subunit ${ }^{4}$, such a mechanism requires that it be possible to move the segment of the polypeptide chain which separates the nucleic acid groove from the lumen of the cylinder so that it is out of the way in the assembly process. Evidence suggesting that the segment in question has some flexibility comes from X-ray diffraction studies of the virus and its components. In the intact virus studied by the Heidelberg group this segment (residues 102-112) apparently exists largely as an $\alpha$ helix (the V-helix) ${ }^{4}$; on the other hand, in the crystals of the isolated coat protein disk studied by the Cambridge group, residues 102-112 and adjacent segments (88-100, 112-114) do not give a well-defined electron density, reflecting a high degree of disorder ${ }^{5}$. However, X-ray evidence is inconclusive on this point, as it does not distinguish between thermal disorder indicating flexibility and static disorder indicating irregular
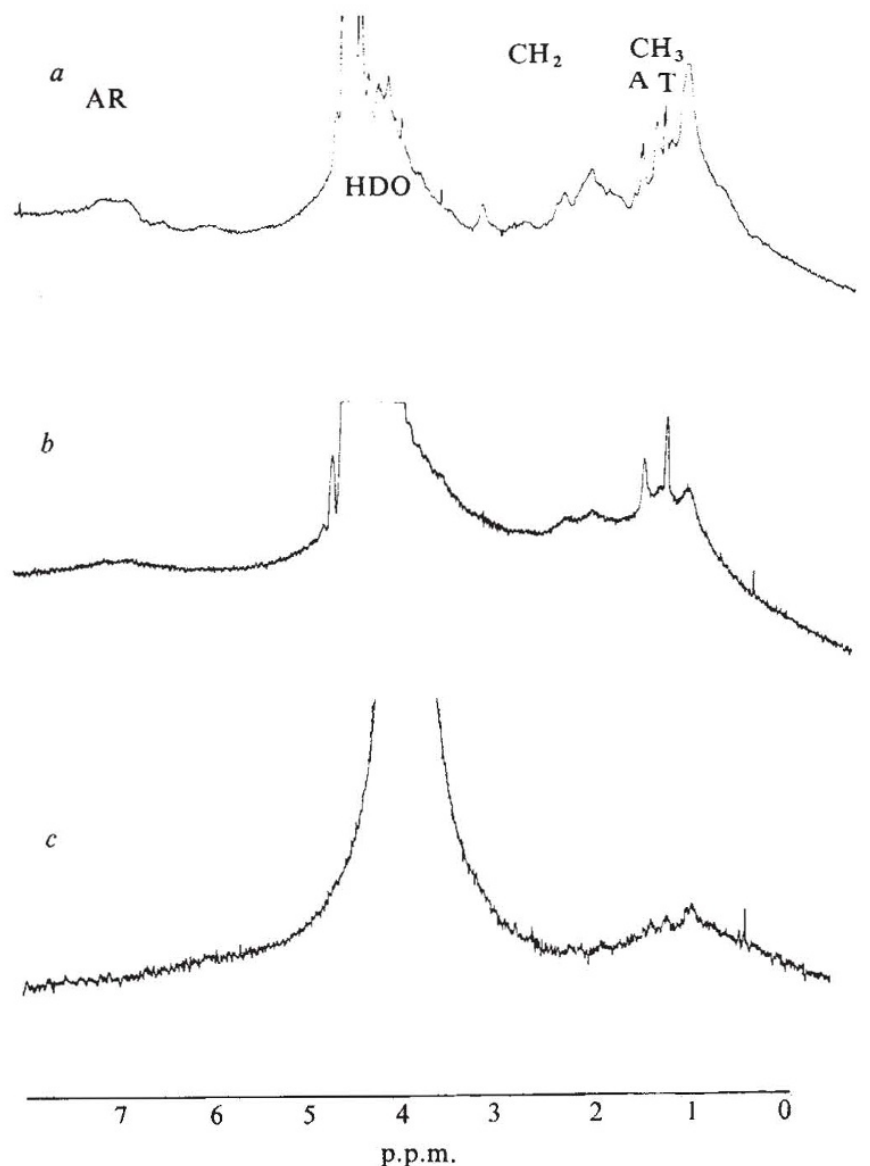

Fig. $1{ }^{1} \mathrm{H}$ NMR spectra of TMV coat protein at $360 \mathrm{MHz}$. Protein concentration $10 \mathrm{mg} \mathrm{ml}^{-1}$ in phosphate buffer $\Gamma / 2=0.1$, temperature $27^{\circ} \mathrm{C}, p \mathrm{H}$ as indicated. $a$, 4S particle, $p \mathrm{H} 8.5 ; b$, disk (19-22S), $p \mathrm{H} \mathrm{7.1;} c$, helix $(\gg 30 \mathrm{~S}), p \mathrm{H}$ 6.0. Chemical shifts $(\delta)$ in p.p.m. from internal DSS. AR = Aromatic envelope; $\mathrm{CH}_{2}=$ methylene envelope, $\mathrm{CH}_{3}-$ methyl envelope; $\mathrm{T}=$ threonine doublet, $\delta=1.17$ p.p.m.; $\mathrm{A}=$ alanine doublet, $\delta=1.42$ p.p.m.

packing of the segment in the crystal. de Wit et al. ${ }^{6.7}$ have pointed out that there must be internal mobility in the TMV coat protein, based on the results of ${ }^{13} \mathrm{C}$ NMR of various TMV protein aggregates. Unfortunately, their results do not show whether or not there is flexibility in a particular segment of the protein. In an effort to distinguish between the thermal disorder and the static disorder in the V-helix segment, we describe here our investigation of the problem by high-resolution proton nuclear magnetic resonance (NMR) studies, and report a flexibility due to thermal motional disorder.

The $360-\mathrm{MHz}^{1} \mathrm{H}$ spectrum of the coat protein (4S particle of TMV Vulgare), average molecular weight (MW) 52,000, as determined by sedimentation velocity on samples used in the NMR studies, is shown in Fig. 1 $a$. The spectrum has several striking features. The aromatic region is surprisingly ill-resolved for a protein of this size, suggesting a relatively well-packed hydrophobic core (ref. 8 and A. Bloomer, personal communication). The aliphatic region is also relatively broad, as might be expected for a protein with a high helical content and tight packing. However, several sharp peaks occur in the 1.1-1.5p.p.m. region downfield from DSS. Most impressive are two sharp doublets at 1.17 and 1.42 p.p.m. The relative sharpness of these lines persists as the effective molecular weight of the coat protein is increased by polymerisation to $8 \mathrm{~S}$ particles, the $19 \mathrm{~S}$ disk (average MW $\sim 650,000$, Fig. $1 b$ ) and to some extent even the RNA-free helix ( $>30 \mathrm{~S}$, MW $>10^{6}$, Fig. $1 c$ ).

There is an obvious possibility that the sharp lines originate from a small molecular weight contaminant. In protein NMR, 
this possibility can sometimes be proved, but seldom rigorously ruled out. Indeed, a third sharp doublet at about 1.3 p.p.m. occurs in some TMV preparations and is readily shown to be a contaminant by the fact that its intensity can be markedly reduced by prolonged dialysis. This is not the case with the two peaks at 1.17 and 1.42 p.p.m. Their absolute and relative intensities are the same in many preparations of TMV Vulgare and several mutants, made in various laboratories, and is not altered by dialysis. Their $\mathrm{T}_{1}$ is of the same order of magnitude as the $T_{1}$ of other protein peaks (compare results below), whereas the $T_{1}$ of the known contaminant is much longer $(\sim 2.4 \mathrm{~s})$. The relative sharpness of these lines is lost on denaturation of the protein, whereas that of the contaminant is not. Even though the evidence is circumstantial, we believe these peaks to represent an integral part of the protein.

From comparison of chemical shifts and coupling constants we conclude that peak $1, \delta=1.17, J \simeq 6.1$, is due to the methyl groups of threonines $(\delta \simeq 1.2, J=6.0-6.6)$ and peak $2, \delta=$ $1.42, J \simeq 6.8$, to the methyl groups of the alanines $(\delta \simeq 1.41$, $J=6.8-7.2$ ). Alternative possibilities, such as unusually shifted methyl groups of valine, leucine or isoleucine, can be excluded by the multiplet structure and double resonance. Peak 1 is found to couple to an $\alpha-\mathrm{CH}$ at 4.32 p.p.m., and peak 2 to one at 4.49 p.p.m., whereas the valine, leucine and isoleucine methyl groups couple to $\alpha-\mathrm{CH}$ groups in the region of $1.5-3$ p.p.m. The relative areas of the two peaks are approximately the same, and comparison with the area of the major peaks in the region 0-3 p.p.m. suggests that they represent three or four threonine and alanine residues each. It is noteworthy that this approximates the threonine and alanine content (four and three, respectively) of the V-helix segment (Ala 100 to Arg 112).

Direct proof that at least some of the sharp lines originate from the V-helix segment comes from an examination of a series of mutants with point amino acid substitutions in the segment. Figure $2 a$ shows a comparison of the spectra of the $4 \mathrm{~S}$ particle of TMV Vulgare, and Fig. $2 b$ the mutant $\mathrm{Ni} 725$, in which Thr 107 is replaced by methionine and Ileu 129 by threonine. Both tracings are obtained by resolution enhancement processing ${ }^{9,10}$, so that the broader components are filtered out and only the sharper components remain. As Vulgare contains no methionine, the assignment of the sharp peak at 2.02 p.p.m. to the methionine methyl group in Ni 725 (ref. 11) is unequivocal. It should be noted that the peak occurring at 2.02 p.p.m. in the tracing in Fig. $2 b$ is actually a superposition of a broader unassigned component present in both spectra, and a sharp methionine peak, which is readily identified in a Hahn-Spin Echo $\mathrm{T}_{2}$ sequence $\left(1 / \pi \mathrm{T}_{2} \sim 4.5 \mathrm{~Hz}\right)$. Less certain are the assignments in the threonine region, as the threonine content of the two proteins is identical. It is probable that the line at 1.2 p.p.m. present in $a$, but not in $b$, represents Thr 107, and the line at 1.2 p.p.m. present in $b$, but not in $a$, represents Thr 129 .

An estimate of the areas of the sharp components ${ }^{10}$ in both spectra also suggests that the peptide segment which shows signs of unusual mobility is longer than the proposed V-helix. Additional peaks with long $\mathrm{T}_{2}$ values occur at 0.8 p.p.m., 1.12 p.p.m., 1.28 p.p.m., 1.35 p.p.m. and 1.6 p.p.m. Except for the alanine and threonine peaks, only four small peaks of Pro 102 and Leu 108 would be expected in this spectral region if only the V-helix contributed the narrow lines. If it is assumed that the entire segment Asp 88-Arg 112 is mobile, the resolution enhanced spectrum predicted from the amino acid composition is in good agreement with that observed. The possibility remains, however, that residues from other segments also contribute to this spectrum and this is being investigated using additional mutants.

Relaxation measurements on the virus coat protein provide further evidence for the interpretation that the protein consists of a relatively compact core containing all the aromatic and most of the hydrophobic aliphatic side chains, and a relatively short, unusually mobile segment.

Our best estimates for the proton relaxation in the aliphatic region are $\mathrm{NT}_{1}=600-700 \mathrm{~ms}, 1 / \pi \mathrm{T}_{2} 10-20 \mathrm{~Hz}$ for the broad components and $\mathrm{NT}_{1}=700-900 \mathrm{~ms}, 1 / \pi \mathrm{T}_{2} 2-4 \mathrm{~Hz}$ for the sharp components. An additional, still broader component for which relaxation measurements have not yet been possible may also exist in the spectrum. Neither of these pairs of measurements can be accounted for by a simple rigid sphere model of relaxation. The relaxation behaviour of the broad component can be approximated by using the 3-state model for internal rotation developed by Woessner ${ }^{12}$, assuming a correlation time $\tau_{c}=2 \times 10^{-8} \mathrm{~s}$ for the overall diffusion of the protein particle, and an average internal correlation time $\tau_{1} \sim 10^{-9}$ for methyl group rotation. However, the model does not account for the behaviour of the sharp component. The theoretical treatment developed by King and Jardetzky ${ }^{13}$ was therefore used to analyse the data for this component. The best fit was obtained using a three-parameter 'Wobble-Woessner' model ${ }^{14}$ with time constant $\tau_{\mathrm{c}}=2 \times 10^{-8} \mathrm{~s}$ for rotational diffusion, $\tau_{\mathrm{s}} \simeq 10^{-8} \mathrm{~s}$ for a $\theta \simeq 90^{\circ}$ segmental wobble and $\tau_{\mathrm{se}} \sim 5 \times 10^{-10} \mathrm{~s}$ for a 3 -state side-chain rotation. The failure of several alternative models to account for the data leads us to believe that the model chosen is a fair approximation to the nature and the rates of the motions involved.

These NMR experiments and others in progress strongly support the notion that the disorder observed in the V-helix segment of the coat protein crystals is of a thermal motional nature. Random coil segments in nucleic acid binding proteins have been noted by Bradbury et al..$^{15}$ in the case of histones. In our experiments, the unusual mobility is not observed by either $\mathrm{X}$-ray or NMR in the intact virus. This suggests that the V-helix

Fig. 2 Resolution enhanced ${ }^{1} \mathrm{H}$ NMR spectra of the 1-2 p.p.m. region, TMV coat protein (4S particles) at $360 \mathrm{MHz}$. $a$, TMV Vulgare; $b$, TMV mutant Ni 725 . Conditions as in Fig. $1 a$.
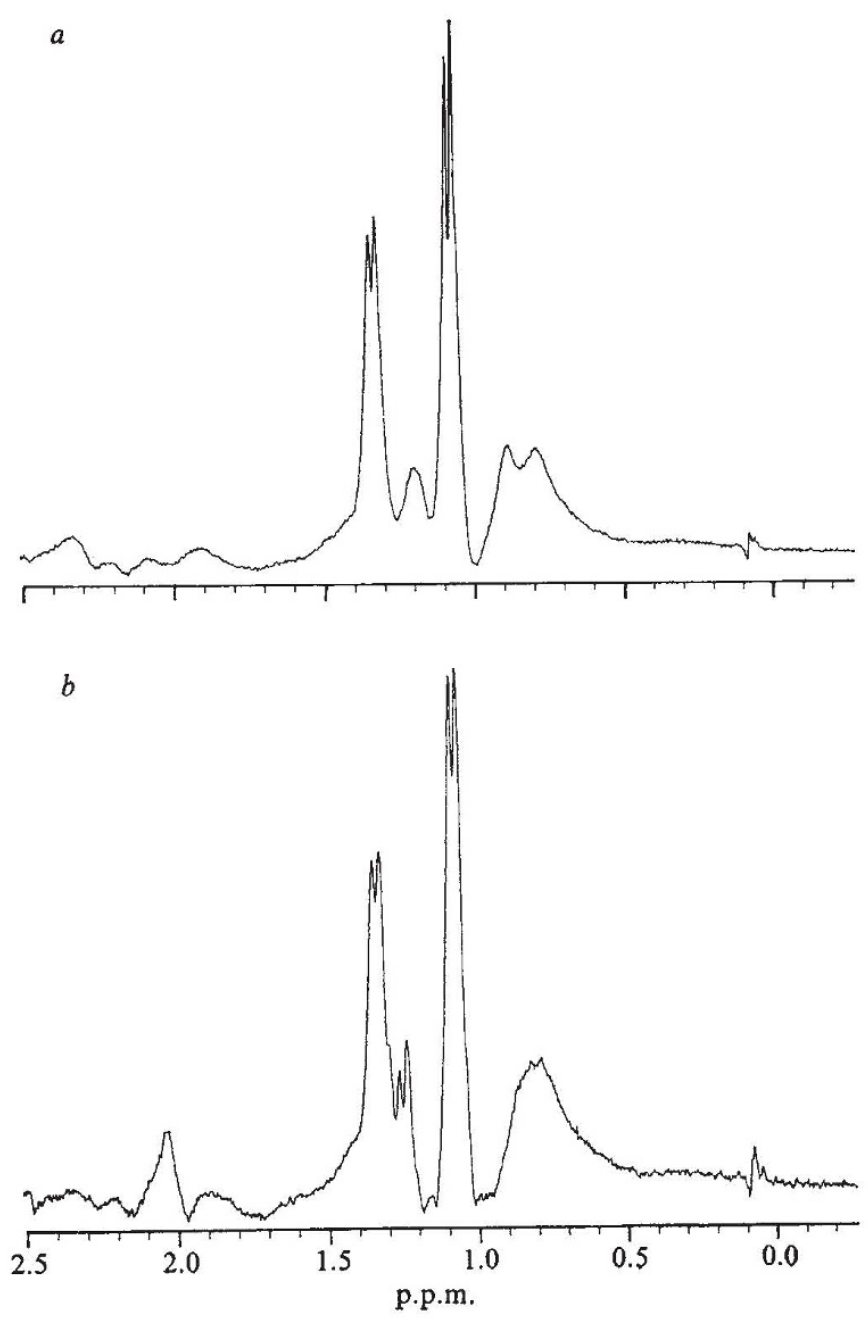
segment acts as a trap door which is open in the RNA-free protein and closed when the nucleic acid is in place.

The experiments reported here are also of interest in another connection. It has recently been claimed ${ }^{16}$ that the relaxation in ${ }^{1} \mathrm{H}$ spectra of globular proteins is dominated by spin diffusion and provides little information on internal motions. This might prove to be true in some cases, but the differential relaxation effects observed here provide a clear example of a protein for which it is not true.

The resolution enhancement processing reported in Fig. $2 a$ and part of the relaxation measurements were carried out on the $360 \mathrm{MHz}$ instrument of the Stanford Magnetic Resonance Laboratory supported by Grant RR00711 from the NIH and Grant GR23633 from the NSF. O.J., on leave from Stanford University, is recipient of a US senior scientist award of the Alexander von Humboldt Foundation, and acknowledges support from NIH Grant GM 18098 and NSF Grant PCM 75-02814. D.V. acknowledges support from a grant of the Deutsche Forschungsgemeinschaft VO $246 / 1+2$. K.A. is on leave from Kyoto University and is a recipient of a fellowship from the Alexander von Humboldt Foundation. The authors thank H. J. Torff for amino acid analysis, Miss U. Gallwitz for technical assistance, and Dr P. Bray and N. Wade-Jardetzky for verifying the findings reported here in a series of experiments to be reported subsequently.

\section{O. JARDETZKY \\ K. AKASAKA}

Department of Molecular Physics,

Max-Planck-Institut für Medizinische Forschung,

Heidelberg, West Germany

Department of Biochemistry,

University of Regensburg, West Germany

D. VoGeL.

S. MORRIS

K. C. Holmes

Department of Biophysics,

Max-Planck-Institut für Medizinische Forschung,

Heidelberg, West Germany

Received 14 October 1977; accepted 7 March 1978.

1. Butler, P. J. G., Finch, J. T. \& Zimmern, D. Nature 265, 217-219 (1977).

2. Butler, P. J. G. \& Klug, A. Nature new Biol. 229, 47-50 (1971).

. Lebeurier, G., Nicolaieff, A. \& Richards, K. E. Proc. natn. Acad. Sci U.S.A. 74, 149-153 (1977).

, S. \& Holmes, K. Nature 267, 216-221 (1977).

. Champness, J. N., Bloomer, A. C., Bricogne, G., Butler, P. J. G. \& Klug, A. Nature 259, 20-24 (1976).

de Wit, J. L., Alma, N. C. M., Hemminga, M. A. \& Schaafsma, T. J. in $N M R$ in Biology (eds Dwek, R. A., Campbell, I. D., Richards, (1977).

7. de Wit, J. L. Hemminga, M. A. \& Schaafsma, T. J. J. Magn. Res (in the press)

Vogel, D. \& Jaenicke, R. Eur. J. Biochem. 61, 423-431 (1975),

Campbell, I. D., Dobson, C. M., Williams, R. J. P. \& Xavier, A. V. Res, 11, 172-181 (1973).

10. Gassner, M. Jardetzky, O. \& Conover, W. W. J. Magn. Res. (in the

press).
1. Wittmann-Liebold, B. \& Wittmann, H. G. \%. Vererhehre 97, 305-326 (1965).

2. Woessner, D. E. J. Chem. Phys, 37, 647-654 (1962)

13. King, R, \& Jardetzky, O. Chem. Phys. Lett. 55, 15-18 (1978).

14. King, $\mathrm{R}$ et al Biophys J (in the press).

15. Chapman, G. E., Hartman, P. G. \& Bradbury, E. M. Eur. I. Biochem. $61,69-75$ (1976)

16. Kalk, A. \& Berendsen, H. J. C. J. Magn. Res. 24, 343-366 (1977)

\section{Multiple regions of metabolic}

\section{activation of carcinogenic hydrocarbons}

Several lines of evidence have strongly implicated the bay region anti-diolepoxide of benzo(a)pyrene (BP) (Fig. 1) as the principal metabolically activated form of this potent environmental carcinogen ${ }^{1}$. A bay region is defined simply as a molecular region such as the 4,5-positions of phenanthrene. Recent studies indicate the bay region diolepoxides of 3-methylcholanthrene (3-MC) (refs 2, 3), benz(a)anthracene ${ }^{4.5}, 7-$ methylbenz(a)anthracene (MBA) (ref. 6), 7,12-dimethylbenz(a)anthracene (DMBA) (refs 7,8$)$, and dibenz $(a, h)$ anthracene ${ }^{9}$ as the ultimate carcinogenic forms of these hydrocarbons. (In the cases of 3-MC, 7-MBA and 7,12-DMBA there is evidence for an additional benzylic hydroxyl group.) Jerina and Daly ${ }^{10}$ suggest, on the basis of simple molecular orbital calculations, that the bay region diolepoxides of carcinogenic polycyclic arenes are generally distinguished by exceptional reactivity and are the ultimately active forms of this class of carcinogen. We report here evidence for metabolites other than bay region diolepoxides as the active forms of at least some carcinogenic hydrocarbons.

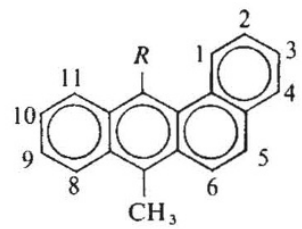

MBA: $R=\mathrm{H}$

DMBA: $R=\mathrm{CH}_{3}$

Metabolic formation of the diolepoxides is believed to proceed by a sequence involving epoxidation, hydration to a transdihydrodiol, and a second epoxidation (Fig. 1). As epoxidation catalysed by the microsomal mixed function oxidases generally fails to occur at substituted aromatic ring positions ${ }^{11}$, it is anticipated that carcinogenic activity should be blocked by substitution in the ring expected to undergo metabolic conversion to a reactive diolepoxide. To test this prediction, we investigated the carcinogenic activity of a series of methyl and fluoro derivatives of BP, MBA, and DMBA substituted in the ring believed to undergo metabolic activation. Our results are summarised in Table 1.

Methyl substitution in the 7- and 10-positions of BP dramatically altered the carcinogenic activity of the parent hydrocarbon. A single intramuscular injection of 1.0 or $2.5 \mathrm{mg}$ of BP induced local sarcoma in $100 \%$ of the male Long-Evans rats tested.
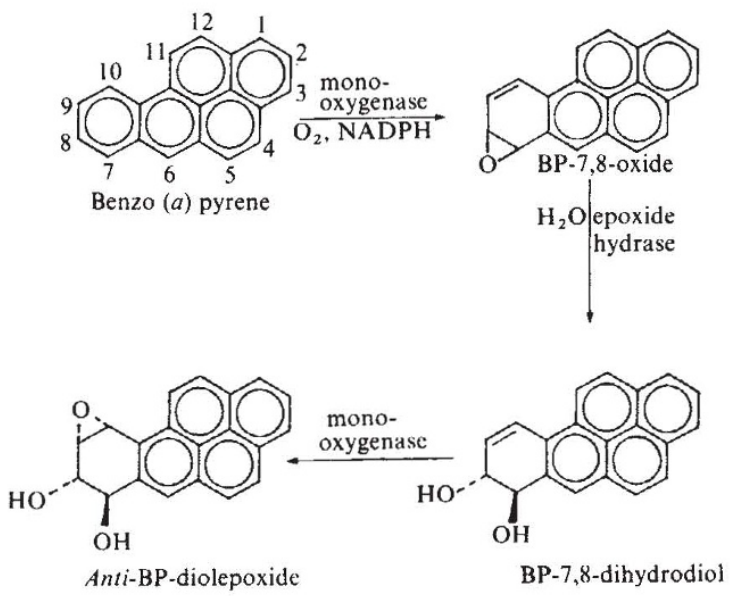

Fig. 1 Metabolic activation of BP catalysed by the microsomal mixed function oxidases.

In contrast, administration of $2.5 \mathrm{mg}$ of 7,10-dimethyl-BP failed to elicit a carcinogenic response in $100 \%$ of animals tested. 10-Monomethyl-BP at similar dosage exhibited very weak activity (one-sixteenth positive), which may not be significant. However, 7-methyl-BP retained a high level of activity, a dose of $2.5 \mathrm{mg}$ inducing sarcoma in seven out of eight rats $(88 \%)$, equivalent at this dosage to that of the carcinogen dibenz $(a, h)$ anthracene. At lower dosage $(1 \mathrm{mg})$ 